\title{
A Pilot Study Evaluating the Development and Implementation of a Feasible, Evidence-based, Group Cognitive Training Program Within an Older People's Mental Health Service
}

Claudia Woolf ( $\square$ claudia.woolf@svha.org.au )

St Vincent's Hospital Sydney https://orcid.org/0000-0003-3214-4765

\section{Danielle Gately}

POWH: Prince of Wales Hospital and Community Health Services

\section{Leanne Kaplan}

St Vincent's Hospital Sydney

\section{Zeeshan Shahnawaz}

St Vincent's Hospital Sydney

\section{Louisa Margaret Norrie}

St Vincent's Hospital Sydney

\section{David Burke}

St Vincent's Hospital Sydney

\section{Sharon Linda Naismith}

The University of Sydney Brain and Mind Research Institute: The University of Sydney Brain and Mind Centre

\section{Loren Mowszowski}

The University of Sydney Brain and Mind Research Institute: The University of Sydney Brain and Mind Centre

\section{Research}

Keywords: cognitive training, mild cognitive impairment, neuropsychology, service delivery, community mental health services

Posted Date: July 26th, 2021

DOl: https://doi.org/10.21203/rs.3.rs-657877/v1

License: (c) (i) This work is licensed under a Creative Commons Attribution 4.0 International License. Read Full License 


\section{Abstract}

Background and objectives

In the absence of a cure for dementia, investigation of novel strategies aimed at delaying or preventing the onset of dementia are gaining momentum. In this regard, using the RE-AIM framework, we outline steps taken to adapt and implement an evidence-based cognitive training program for older adults 'at risk' of developing dementia within a clinical setting of a major metropolitan hospital. The primary aim was to explore feasibility (or 'reach'), tolerability (or 'implementation'), and acceptability (or 'adoption'). The secondary aim was to explore the most sensitive clinical outcomes and measurement tools (i.e. 'efficacy') to inform a subsequent formal feasibility randomised controlled trial.

Method

This was an exploratory feasibility pilot study, using a pre-post single-arm study design. We conducted this study within the Older People's Mental Health Service at St Vincent's Hospital, Sydney, Australia. Seventy-nine participants, with a mean age of 78 years $(S D=5.8$, range $=67-94)$ were enrolled. All enrolled participants engaged in 'Club Connect' an adaptation of a 10-week, group-based multifaceted program, comprised of psychoeducation and computer-based cognitive training. At baseline and follow-up, all participants completed a battery of neuropsychological tests and several questionnaires related to mood and psychosocial functioning.

\section{Results}

Implementing cognitive training within a clinical setting was feasible, well tolerated and accepted by participants. Further, cognitive training was associated with large to very large effect size improvements in verbal learning, memory for verbal and visual information, and semantic fluency, as well as quality of life, and reductions in anxiety and stress. We also found small to medium effect size improvements for other cognitive and psychosocial outcomes.

\section{Conclusions}

Our preliminary findings support the feasibility of translating a group-based cognitive training program into the clinical setting, and provide key data to facilitate the next phase of implementation.

\section{Contributions To The Literature}

- Cognitive training offers promise as a novel treatment intervention for older adults who are 'at-risk' of developing dementia. Yet, while considerable consumer and provider enthusiasm exists, there remains a significant 'evidence-to-practice' gap, with very few cognitive training interventions being offered in the clinical setting.

- Our findings highlight an urgent need for further translational research that evaluates the implementation and effectiveness of CT in everyday clinical practice, which will in turn provide stakeholders and decision 
makers with rich and reliable data to guide implementation decisions, ultimately improving access to innovative, evidence-based mental health care.

\section{Introduction}

Dementia is the second leading cause of death in Australia and the single greatest cause of disability among older Australians [1]. However, to-date, pharmacological treatments for dementia are aimed at symptom reduction in the absence of a cure. Therefore, investigation of novel strategies aimed at delaying or preventing the onset of dementia is gaining momentum.

In this regard, cognitive remediation is one avenue that holds promise in 'at-risk' ageing populations. Cognitive remediation refers to a broad range of therapeutic approaches designed to ameliorate cognitive deficits. Efforts to clarify this range of techniques have identified three primary approaches: cognitive stimulation, cognitive training (CT) and cognitive rehabilitation [see 2]. Of these, CT is gaining increasing prominence due to its potential to maintain and enhance cognition, and improve psychosocial functioning. CT programs enhance cognition by providing theoretically driven 'guided practice' on various tasks reflecting different cognitive functions. CT may be strategy-based, incorporating both internal and external compensatory cognitive strategies, or computer-based, incorporating drill-and-practice exercises targeting specific cognitive domains $[2,3]$.

There is now a solid body of evidence demonstrating efficacy of CT for improving cognition in psychiatric populations including schizophrenia [4] and major depression [5], in healthy older adults [6], and in groups at risk of developing dementia, i.e. those with mild cognitive impairment $(\mathrm{MCl})[2,3,7,8]$. Meta-analytic studies in $\mathrm{MCl}$ have demonstrated moderate to large effect size improvements for memory (Hedges $g=0.3-0.5)[9$, 10], with small to large effects (Hedges $g=0.2-0.7$ ) demonstrated for attention, processing speed and executive functioning $[9,10]$. Additionally, several groups investigating $\mathrm{CT}$ in $\mathrm{MCl}$ and healthy older adult cohorts have reported generalised gains in psychosocial and functional domains, such as improved performance in simulated real-life tasks [11], improved mood and sleep [8, 12], informant-rated activities of daily living [12] and increased knowledge and use of memory strategies in everyday life as well as improved self-efficacy $[13,14]$. There is also growing evidence that these benefits are sustained several months or even years following CT $[15,16]$. Importantly, one consistent finding across studies is the excellent adherence, tolerability and lack of negative effects of CT [17], unlike pharmacological interventions which often carry significant adverse side effects.

Despite this, there unfortunately remains an evidence-to-practice 'implementation gap', with few CT programs being offered in public health settings. For example, in Australia, a recent survey showed that a minority of memory clinics offered interventions [18], and of those offered, only one session was provided. There are some studies of CT implementation in those with psychosis or schizophrenia [19-21], serious mental illness [22], depression [23, 24] and $\mathrm{MCl}[13,25]$; however, the evidence base is very limited and few studies report on key elements of research translation. Those that do report on these elements seldom incorporate their findings within an overarching 'implementation framework', which increases the challenge in interpreting results across studies. As an example, key research translation factors are addressed within the RE-AIM framework comprised of five evaluation dimensions (Reach, Effectiveness, Adoption, Implementation, 
Maintenance), that can be used to comprehensively evaluate both the individual and organisational impact of an intervention [26]. Researchers must more readily recognise the crucial need to perform formative evaluations assessing the extent to which implementation is effective in a specific context to: optimise intervention benefits, prolong sustainability of the intervention in that context, and promote dissemination of findings into other contexts [27, 28]. At this stage, neither the RE-AIM framework nor previous studies of CT implementation set specific, quantitative limits to define what should be expected with regard to feasibility of trial design, tolerability and acceptability.

Understanding how to make CT programs accessible and feasible in clinical populations for individuals 'at risk' of developing dementia is complex), yet ultimately, this should represent the end goal of the translational research framework. In this chapter, using the RE-AIM framework [26], we outline initial steps taken to adapt the evidence-based, multifaceted psychoeducational and CT 'Healthy Brain Ageing' program, and implement it, for the first time, as "Club Connect", into a clinical psychogeriatric setting. The primary aim for this pilot study was to explore feasibility (recruitment rates; 'reach'), tolerability (adherence to treatment protocol; 'implementation'), and acceptability (of data collection procedures and the intervention; 'adoption') of Club Connect in a metropolitan Older People's Mental Health (OPMH) Service. The secondary aim was to explore optimally sensitive clinical outcomes and measurement tools ('efficacy') to inform a subsequent formal feasibility RCT.

\section{Methods}

\section{Procedure for research translation}

After an initial meeting between key stakeholders from the Brain and Mind Centre (BMC) at The University of Sydney (SN, LM) and those from the OPMH Service (CW, LN) at St Vincent's Hospital (SVH), a formal agreement was made to implement the Healthy Brain Aging (HBA) program within the OPMH Service.

The HBA CT program was developed for help-seeking older adults 'at risk' of cognitive decline [see 8, 29]. Conducted in a group format, it comprises of a psychoeducational and cognitive strategy component (50 minutes), with a brief tea break to facilitate socialisation, followed by computerised CT (50 minutes), utilising the Neuropsychological Educational Approach to Remediation (NEAR) [30]. Evaluations of the program, including RCTs, have demonstrated improvements in memory and dementia literacy in those with late-life depression (Naismith et al., 2011; Norrie et al., 2011) and in memory, depressive symptoms, and sleep quality in $\mathrm{MCl}$ (Diamond et al., 2015; Mowszowski et al., 2014), and improvements in memory in Parkinson's disease (Naismith et al., 2013). Importantly, across these samples, participants were on average 65 years old with $>13$ years of formal education.

The first step in implementing the HBA program within SVH OPMH involved training from the HBA clinical lead (LM) in: psychoeducation material, logistical resources (e.g. session plans), face-to-face training in computer-based CT techniques and NEAR principles, and observation of the HBA program at BMC.

The HBA program was re-branded to 'Club Connect' in line with other group-based interventions within OPMH. The clinical lead of the HBA program attended several sessions of Club Connect in the early stages to ensure 
program fidelity within the local context. Ongoing clinical supervision was also provided. An iterative approach to adapting the program for OPMH was taken; therefore, our methods and results will be reported here as two sequential studies.

The Club Connect team comprised one 'champion' clinician, a Clinical Neuropsychologist (CW), and supporting clinicians including a Clinical Neuropsychologist (LK); Clinical Psychologist (ZS), Psychogeriatrician (LN) and Occupational Therapist (DG).

\section{Study 1:}

\section{Method}

\section{Participants}

Commencing in October 2015, 25 participants were recruited from OPMH or Geriatric Medicine at SVH. Referrals were received via the OPMH intake officer, and discussed with a member of the Club Connect team at the time of referral and again at the weekly OPMH case review meeting. Participants were recruited according to criteria placing them 'at risk' of cognitive decline including having i) subjective cognitive impairment or ii) an existing diagnosis of $\mathrm{MCl}$, and were required to be aged 65 years or above, and be willing to attend 10 weekly sessions of CT. Exclusion criteria were: i) psychiatric illness other than an affective disorder and ii) a diagnosis of dementia with functional impairment. Written consent was obtained from all participants. The study was approved by SVH Human Research Ethics Committee.

\section{Design}

This was an exploratory feasibility study, using a pre-post, single arm study design. Allocation depended on availability at the time of referral. At baseline, all participants completed a battery of neuropsychological tests and a number of questionnaires related to psychosocial functioning within the fortnight prior to the intervention commencing (i.e. weeks $0-2$ ). These assessments were repeated at follow-up, within a fortnight of the intervention ceasing (i.e. weeks 13-14). See Figure 1 for a timeline of study procedures.

Study 1 was conducted across two recruitment 'waves'; the first wave $(n=15)$ was completed from October to December 2015 and the second wave $(n=10)$ was completed from April to July 2016.

\section{Measures}

All participants completed:

- the Repeatable Battery for the Assessment of Neuropsychological Status (RBANS) [31],

- the Frontal Assessment Battery (FAB) [32],

- the Geriatric Depression Scale 15-item (GDS-15) [33],

- the Depression Anxiety and Stress Scale (DASS-21) [34],

- the World Health Organisation Quality of Life-BREF (WHOQOL-BREF) [35],

- the WHO Disability Assessment Scale 2.0 (WHO-DAS 2.0) [36], 
- the HBA Functional Assessment Questionnaire,

- the Memory Complaint Questionnaire (MAC-Q) [37],

- the Prospective and Retrospective Memory Questionnaire [38],

- At follow-up, an adapted version of the HBA feedback form was administered to gauge participant satisfaction and obtain feedback regarding program content, format and service delivery.

(for a full description of the above, please see Additional File 1).

\section{Outcomes}

The primary outcome of this study was feasibility, defined as:

- Feasibility, or 'reach': recruitment rates and/or strategy i.e. number/percent of referrals eligible and consented.

- Tolerability, or 'implementation': adherence to treatment protocol i.e. number/percent of sessions attended over the intervention period and number/percent of participants who completed baseline and follow-up assessments.

- Acceptability,or 'adoption': of data collection procedures and the intervention, as perceived by patients, measured using the adapted HBA evaluation form.

- Clinical outcomes for 'efficacy': explore (effect size) improvements to determine the most appropriate and sensitive clinical outcome tools to inform a future full-scale feasibility RCT.

\section{Intervention}

The Club Connect intervention followed the original HBA 10-session, two-hour weekly group format [see 29]. It included i) 50 minutes of psychoeducation and ii) 50 minutes of computer-based CT, with a 15-20 minute break between components where refreshments were provided and clinicians left the room to facilitate peer socialisation.

1. Psychoeducation: this component included semi-structured PowerPoint presentations on the following topics: the brain, attention and processing speed, learning and memory, executive function, vascular risk factors, diet and exercise, depression and anxiety, and sleep. All material was delivered by multidisciplinary specialists (Clinical Neuropsychologists, Clinical Psychologists, Old Age Psychiatrists etc.) in the relevant field and in accordance with scientific literature. This component had a maximum of 14 participants per group.

2. CT: the CT intervention was delivered by two clinicians, typically a Clinical Neuropsychologist and a Clinical Psychologist or Occupational Therapist, and all utilised the NEAR approach [30]. Participants followed an individualised computer-based CT program devised according to their baseline neuropsychological strengths and weaknesses, drawing from a range of programs comprising educational software and commercially available CT programs. This component had a maximum of seven participants per group. 
Analyses were conducted using SPSS Version 25. Baseline data was summarised using means (with standard deviations), counts and proportions. The proportion of appropriate referrals of eligible and consenting participants (i.e. feasibility), and adherence to treatment protocol (i.e. tolerability) was calculated. Acceptability was analysed qualitatively and using frequencies. One-way repeated measures ANOVA with effect sizes (i.e. partial eta squared) and 95\% confidence intervals was used to explore differences in participants' performance on all clinical outcomes before and after the 10-week intervention.

\section{Results}

\section{Descriptive sample characteristics}

Twenty-five individuals were referred to Club Connect comprising two waves of recruitment, intervention and data collection. The average age of participants was 78.2 years ( $S D=5.6$; range 69-92), and the majority (72\%) were male. With regard to education, $24 \%$ had 12 years or less of formal education, $44 \%$ had $13-15$ years of formal education, and $32 \%$ had 16 or more years of formal education. Retrospective data on cognitive screening tools was available for 23 of the 25 participants; 14 participants completed the Montreal Cognitive Assessment and the mean score was 23.83 (SD: 2.7, range: 20-29), and nine participants completed the Mini Mental State Examination (MMSE) and the mean score was 27.89 (SD: 1.83, range: 2530).

\section{Feasibility}

All 25 participants referred to Club Connect were eligible and consented to participate; therefore the trial design was considered feasible. 24 participants completed the program (i.e. attended both baseline and follow-up assessment). The participant that did not complete Club Connect was bereaved during the course of the program.

\section{Tolerability}

The intervention was well-tolerated by participants, as $96 \%$ attended $\geq 7$ of the 10 sessions; $72 \%$ attended $\geq 9$ sessions.

Data collection procedures were also well-tolerated by participants. At baseline, all 25 participants completed cognitive assessment, $96 \%$ completed mood questionnaires and $84 \%$ completed the psychosocial questionnaires. All 24 'completers' underwent cognitive and mood assessment at follow-up, and 92\% completed psychosocial questionnaires at follow-up.

\section{Acceptability}

Sixteen individuals anonymously completed the evaluation survey, although some participants did not respond to all items. Regarding the psychoeducation material, 88\% of responders reported that the education sessions helped them understand what affects thinking skills in older age; all responders reported that they use the strategies to improve their day-to-day activities, and 87\% reported that they planned to make changes to their lifestyle based on the psychoeducation provided. Regarding the computer-based CT component, on a 
scale of 1 to 10 (where 1 represented 'not at all' and 10 represented 'very much'), $77 \%$ of responders selected a score of 8 or greater in response to 'how much did you enjoy working on the computers?' When asked about the likelihood of continuing to use the computer programs at home, $64 \%$ responded "definitely" and $29 \%$ responded "maybe", with only one participant responding "no". All responders reported that they would recommend 'Club Connect'.

Qualitative responses on the evaluation survey indicated a general sentiment that the program was suitable and acceptable to their needs (e.g. "I enjoyed every moment") and that it should continue in the future (e.g. "keep going, congratulations"; "continue the way you all have over the last 10 weeks"). Suggested improvements fell into three categories; a) additional resources, b) content improvements, and c) delivery improvements. Additional resources included multiple requests for a refresher course. Content improvements included changes to the computer games including more Australian-specific options. Delivery improvements included simplification of language used in the psychoeducation sessions.

\section{Clinical outcomes}

Several one-way repeated measures ANOVAs were conducted to determine if there were improvements on a range of clinical outcomes following participation in Club Connect (see Table 1). Results indicated statistically significant mean differences, with large to very large effect sizes on RBANS List Learning, $F(1$, $23)=4.95, p=0.036, \eta p^{2}=0.177$, RBANS Semantic Fluency, $F(1,23)=13.72, p=0.001, \eta p^{2}=0.374$, WHOQOLBREF Psychological Domain, $F(1,15)=5.70, p=0.031, \eta p^{2}=0.374$, DASS Anxiety Scale, $F(1,22)=9.52$, $p=0.05, \eta p^{2}=0.302$, and DASS Stress Scale $F(1,22)=5.14, p=0.034, \eta p^{2}=0.189$. Small, medium and large effect sizes were evident on other cognitive and psychosocial measures (see Table 1).

\section{Study 2}

Given that refinement based on iterative feedback is a critical component of implementation science and central to the RE-AIM framework [39], we used our own learnings as well as participant feedback from Study 1 to further refine and adapt the intervention to our setting. This iteration and it's evaluative analysis are described here as Study 2.

In response to participant feedback from Study 1, we simplified the language used for the psychoeducation material, and we also slightly amended the psychoeducation schedule (i.e. instead of three memory sessions and one executive function session, participants in Study 2 received two memory and two executive function sessions). Additionally, we amended the assessment battery in response to feedback from clinicians that the existing neuropsychological battery did not sufficiently assess executive function or psychosocial functioning, and did not include a medical review. In addition to this, further amendments to the assessment battery were made through the course of Study 2 (as indicated by 'third wave only' in the 'Measures' section below) to provide even further assessment of executive function and to more sensitively assess activities of daily living, as well as to provide assessment of wellbeing and cognitive stimulation. All amendments to the study protocol were approved by SVH HREC.

\section{Methods}




\section{Participants}

Fifty-five participants were recruited from OPMH or Geriatric Medicine at SVH using the same recruitment methods as in Study 1. In addition to existing inclusion criteria, these participants were also required to score MMSE>23 at baseline. Written informed consent was obtained from all participants.

\section{Design}

The design for Study 2 was identical to that of Study 1. Study 2 was completed across three recruitment waves: the first wave $(n=22)$ was completed from September to December 2016, the second wave $(n=17)$ was completed from February to May 2017, and the third wave $(n=16)$ was completed from August to November 2017.

\section{Measures}

Medical: An Old Age Psychiatrist (LN, KM, JH) or an Advanced Trainee in Old Age Psychiatry (YS) assessed all participants at baseline and administered:

- the Cumulative IIIness Rating Scale (Geriatric version) (CIRS-G) [40],

- the Major Depressive Episode, Dysthymia, Suicidality, Manic and Hypomanic Episode, and Generalised Anxiety Disorder subscales from the Mini International Neuropsychiatric Interview (MINI) [41],

- the Hamilton Depression Rating Scale (HAM-D) [42].

Cognitive: a brief neuropsychological assessment conducted by a Clinical Neuropsychologist (CW, LK) and included:

- Coding from the Wechsler Adult Intelligence Scale-Fourth Edition (WAIS-IV) [43],

- Controlled Oral Word Association Test (COWAT) [44],

- RBANS Figure Copy and Figure Recall [31],

- Logical Memory (LM) from the Wechsler Memory Scale-Fourth Edition (WMS-IV) [43],

- Hopkins Auditory Verbal Learning Test (HVLT) [45],

- Similarities from the WAIS-IV [43],

- Colour Word Interference Test from the Delis-Kaplan Executive Function System (D-KEFS) [46],

- Trail Making Test (TMT-A and B, seconds) [47] (third wave only).

Alternate forms were used, where possible.

Psychosocial:

- Mood:

1. GDS-15 [33],

2. DASS-21 [34],

3. Geriatric Anxiety Scale (GAS) [48], 
- Sleep: The Pittsburgh Sleep Quality Index (PSQI) [49],

- Quality of life: WHO-QoL BREF [35].

- Function:

1. The OARS Multidimensional Functional Assessment Questionnaire [50] (wave one and two),

2. The HBA Functional Assessment Questionnaire (third wave only),

- Wellbeing: The WHO Wellbeing Index [51] (third wave only),

- Cognitive stimulation: HBA Cognitively Stimulating Activities Questionnaire (third wave only),

- Feedback: adapted version of the HBA evaluation form at follow-up.

(for a full description of the above, please see Additional File 2).

\section{Intervention}

As described above, the psychoeducation material and schedule were amended for Study 2. The intervention was otherwise identical to Study 1 .

\section{Outcomes}

Feasibility outcomes for Study 2 were identical to Study 1 .

\section{Statistical analysis}

Statistical analyses for Study 2 were identical to those described for Study 1.

\section{Results}

\section{Descriptive sample characteristics}

Fifty-five individuals were referred to Club Connect. The average age of participants was 77.90 years ( $S D=5.9$; range $67-94$ ); just over half (54.5\%) were female; $36 \%$ had $\leq 12$ years of formal education, $21.8 \%$ had $13-15$ years of formal education, and $41.8 \%$ had $\geq 16$ years of formal education. The mean MMSE score was 27 $(S D=1.6$; range 24-30), representative of a non-demented cohort; the mean HAM-D score was $4.85(S D=4.28$; range 0-15), indicative of a cohort with normal depressive symptoms; and the mean of the total score on the CIRS-G was 8.06 (SD=4.72; range 0-20) (no severity index provided for the total score).

\section{Feasibility}

Of the 55 participants referred to Club Connect, 54 were enrolled. Of these, $80 \%$ were 'completers', defined as having completed both baseline and follow-up assessments. Since $98 \%$ of referred participants were enrolled, and $80 \%$ of participants completed the program, the trial design was considered to be feasible. For those who did not complete the program, this was due to: a) deterioration in physical or mental health $(n=6), b)$ preexisting arrangements (e.g. scheduling conflicts, planned holidays or caregiving) $(n=4)$, and c) being unsuitable for the program due to chronic health conditions (end-stage Parkinson's disease $(n=1)$ and narcissistic personality disorder $(n=1))$. 


\section{Tolerability}

The intervention was well-tolerated by participants as $80 \%$ attended $\geq 7$ sessions and $54 \%$ attended $\geq 9$ sessions.

Data collection procedures were also considered to be well-tolerated by participants. At baseline, of the 54 participants who were eligible and enrolled, $98 \%$ completed cognitive assessment, $95 \%$ completed mood questionnaires and $95 \%$ completed the psychosocial questionnaires. Missing data for one participant was due to being inappropriate for the group (due to a narcissistic personality disorder) which was determined during baseline assessment. Other missing data at baseline was due to data inadvertently not being collected/stored. Of the 43 'completers' at follow-up, 79\% completed cognitive assessment, and 88\% completed both the mood and psychosocial questionnaires. In some $(n=6)$ cases, follow-up cognitive assessments were deliberately omitted (to limit exposure to neuropsychological test material) as these participants were due to have formal neuropsychological assessment at $\mathrm{OPMH}$, to inform their ongoing clinical management.

\section{Acceptability}

Forty-two of the 43 completers anonymously completed the evaluation survey, although some participants did not respond to all items. In regard to the psychoeducation material, $95 \%$ of participants reported that the education sessions helped them to understand what affects thinking skills in older age, all reported that they use the strategies to improve their day-to-day activities, and $95 \%$ reported that they would make changes to their lifestyle based on the psychoeducation provided. In regard to the computer-based CT, on a scale of 1 to 10 (where 1 represented 'not at all' and 10 represented 'very much'), 76\% of participants provided a score of 8 or greater in response to 'how much did you enjoy working on the computers?', and $83 \%$ indicated that they would 'definitely' use the computer programs at home after Club Connect finished. All participants reported that would recommend the Club Connect program.

Qualitative responses to the evaluation survey indicated a general sentiment that the program was suitable and acceptable, and that it should continue into the future (e.g. "no improvement is necessary, stick with such an excellent program"; "more than satisfied with it - only sorry when it ended... it was good to meet the participants and to feel less 'alone'..."). Suggested improvements fell into three categories; a) additional resources, b) content improvements, and c) delivery improvements. Additional resources again included requests for a refresher course, access to videos of the sessions to re-visit content, and a method for keeping in contact with other members once the program ended. Content improvements included changes to computer games (e.g. more options and more Australian-specific options), expanded lecture content (grief, trajectory of dementia), and adding a glossary of terms to the workbook. Delivery improvements included adaptations for audio and/or visual impairment, further simplification of psychoeducation language, inclusion of real-life examples, and encouraging greater participant interaction during lectures.

\section{Clinical outcomes}

Several one-way repeated measures ANOVAs were conducted to determine if there were improvements on clinical outcomes (see Table 2). Results demonstrated statistically significant mean differences, with very 
large effect sizes on HVLT delay, $F(1,31)=10.167, p=0.003, \eta p^{2}=0.247$ and RBANS Figure Recall $F(1,26)=$ $14.315, p=0.001, \eta p^{2}=0.355$. Small, medium and large effect sizes were evident on other cognitive and psychosocial measures (see Table 2).

\section{Discussion}

This pilot study aimed to evaluate the iterative adaptation and implementation of the evidence-based HBA group CT program, re-branded as Club Connect, within OPMH at SVH. Unlike existing studies of CT implementation that seldom perform formative evaluations of research translation within an 'implementation framework', we used the RE-AIM framework to evaluate feasibility ('reach'), tolerability ('implementation'), and acceptability ('adoption').

Our preliminary findings, consistent with other studies of CT in public health settings [13, 19, 21-23, 25], supports the feasibility of translating group-based CT into clinical practice. Regarding feasibility (or 'reach'), across Studies 1 and 2, given all participants referred were eligible, and all but one participant was enrolled, the overall trial design was considered feasible. Findings from a recent meta-analysis of 18 studies of computerised CT in adults aged $\geq 55$ years with $\mathrm{MCl}$ demonstrated drop-out rates of $0 \%$ to $32 \%$ [9]. Given that our cohort comprised a clinical population, where higher rates of non-participation and discontinuation may be expected, it is reassuring that our attrition rate was only $20 \%$, which is considerably less than attrition rates reported in other studies of CT implementation [19, 20,22, 23]; except for one study, in $\mathrm{MCl}$, where an attrition rate of only $5 \%$ was recorded [25]. Unsurprisingly and consistent with other studies of CT implementation [19, $20,25]$, acute illness, both physical and mental, was reported as the primary reason for dropout.

Regarding tolerability of the intervention, or 'implementation', across Studies 1 and 2, the mean attendance rate was $9.0(S D=1.05)$ sessions, suggesting that the intervention was well-tolerated by participants. This $90 \%$ attendance rate is relatively consistent with our previous work in the research setting [8] and the one other known study of $\mathrm{CT}$ implementation in $\mathrm{MCl}$ (where all participants attended all 12 sessions) [25], and certainly higher than that reported in studies of CT implementation in schizophrenia of $42.5 \%$ [21]), psychosis of $72 \%$ [19], and depression of 68 to $84 \%[23,24]$. However, attendance rates are undoubtedly affected by the unique characteristics and considerations of different patient cohorts.

We also examined tolerability of data collection procedures or 'implementation', recognising that program evaluation needs to strike a balance to avoid over-burdening whilst ensuring that methods are thorough enough to be meaningful. Across Studies 1 and 2, 79\% of enrolled participants were 'completers' (i.e. completed data collection at baseline and follow-up). This is consistent with other studies of CT implementation, where full data collection rates are reported between $48-66 \%$ in schizophrenia and serious mental illness [20-22], 95\% in depression [23, 24], and 100\% in $\mathrm{MCl}$ (albeit in a single, small sample $(n=19)$ study) [25]. However, comparison of these figures should be interpreted with caution given the time required to complete assessment likely varies across studies, as does the patient group.

Regarding acceptability or 'adoption', $100 \%$ of participants who completed the program reported that they would recommend Club Connect to their peers, demonstrating overwhelming acceptability of the program. Our rates are somewhat higher than is reported by Eigenhuis, Seldenrijk (24) of CT implementation in 
depression, where $85 \%$ of participants report they would recommend the program; however these lower rates may relate to greater depressive symptomatology in their sample. Qualitative feedback indicated a general sentiment from participants that the program was suitable and acceptable, and that it should continue into the future. This is particularly important from a systems perspective, in relation to advocating for changes in service provision. Surprisingly, of the existing studies of CT implementation, there is sparse assessment of 'acceptability' which is concerning [26].

As a secondary aim, we sought to explore the most sensitive clinical outcomes to explore 'efficacy' in the clinical setting and to inform a larger, feasibility RCT. Across Studies 1 and 2, statistically significant mean improvements, with large to very large effect size improvements, were demonstrated on several cognitive tests including verbal learning, verbal and non-verbal memory, and semantic fluency, as well as on the psychological domain of quality of life, and the anxiety and stress scales of the DASS. Across Studies 1 and 2 we found small to medium effect size improvements for most other cognitive and psychosocial outcomes. These preliminary results are consistent with our prior work [8, 29,52], and with other CT studies in 'at risk' older adults [10]. We acknowledge that the absence of a control group limits our capacity to draw definitive conclusions regarding efficacy; nonetheless, the direction and magnitude of effects shown here and their consistency with other research-based studies are encouraging. At the very least, these findings indicate that the outcome measurement tools used in both studies were sufficiently sensitive to detect change postintervention.

The main limitations of this study are inherent to the pilot design - conclusions are tentative until confirmed by a full-scale trial. This included the absence of a control condition and lack of blinded evaluators.

Additionally, the contribution and severity of cognitive and depressive symptoms, the level of motivation, and concurrent psychosocial and pharmacological treatments were not evaluated or controlled for in the current study. Further, the current study did not evaluate the 'acceptability' or 'adoption' of the program among clinicians. In relation to facilitator training, we used a 'train the trainer' model as a pragmatic approach to training, although we did not systematically examine fidelity in the facilitators' implementation of the program. Finally, a formal economic analysis was not included in the current study even though previous studies suggest that cognitive remediation programs may result in service cost savings [53]. All of the aforementioned should be addressed in a full-scale trial.

\section{Conclusion}

This study supports the feasibility of implementing a group, evidence-based CT program within a metropolitan OPMH Service, given it was well tolerated and accepted by participants 'at risk' of cognitive decline, while being flexible to local adaptions as evaluated by the RE-AIM framework. Our results, in light of the recognised 'evidence to practice gap', which sees few interventions being made available for managing cognitive impairment in older adults, highlights an urgent need for further translational research to evaluate the implementation and effectiveness of CT in everyday clinical practice, which together with greater political and community acceptance for the concept of proactive and 'positive ageing', will help build an argument for public health funding for cognitive interventions for community-based older adults. 


\section{Declarations}

\section{Ethics approval and consent to participate}

Included on page 8.

\section{Consent for publication}

Not applicable.

\section{Availability of data and materials}

The datasets used and/or analysed during the current study are available from the corresponding author on reasonable request.

\section{Competing interests}

The authors declare that they have no competing interests.

\section{Funding}

The research was supported by grant funds from the St Vincent's Clinic Foundation.

\section{Authors' contributions}

CW contributed to study design, collected data, facilitated the intervention, was responsible for statistical analyses and wrote the manuscript. DG collected data and facilitated the intervention. LK collected data and facilitated the intervention. ZS contributed to study design, collected data and facilitated part of the intervention. LN contributed to study design, collected data and provided clinical governance. DB contributed to study design and provided clinical governance. SLN contributed to study design and assisted with writing the manuscript. LM contributed to study design, provided clinical supervision and assisted with writing the manuscript.

\section{Acknowledgements}

Not applicable.

\section{References}

1. Australian Institute of Health and Welfare. Dementia 2020 [Available from: www.aihw.gov.au/reports/australians-health/dementia.

2. Mowszowski L, Batchelor J, Naismith SL. Early intervention for cognitive decline: can cognitive training be used as a selective prevention technique? International psychogeriatrics. 2010;22(4):537-48.

3. Gates NJ, Sachdev PS, Fiatarone Singh MA, Valenzuela M. Cognitive and memory training in adults at risk of dementia: a systematic review. BMC geriatrics. 2011;11:55. 
4. Wykes T, Huddy V, Cellard C, McGurk SR, Czobor P. A meta-analysis of cognitive remediation for schizophrenia: methodology and effect sizes. The American journal of psychiatry. 2011;168(5):472-85.

5. Woolf C, Lampit A, Shahnawaz Z, Sabates J, Norrie LM, Burke D, et al. A Systematic Review and MetaAnalysis of Cognitive Training in Adults with Major Depressive Disorder. Neuropsychol Rev. 2021.

6. Lampit $\mathrm{A}$, Hallock $\mathrm{H}$, Valenzuela $\mathrm{M}$. Computerized cognitive training in cognitively healthy older adults: a systematic review and meta-analysis of effect modifiers. PLoS medicine. 2014;11(11):e1001756.

7. Gavelin HM, Lampit A, Hallock H, Sabates J, Bahar-Fuchs A. Cognition-Oriented Treatments for Older Adults: a Systematic Overview of Systematic Reviews. Neuropsychol Rev. 2020;30(2):167-93.

8. Diamond K, Mowszowski L, Cockayne N, Norrie L, Paradise M, Hermens DF, et al. Randomized controlled trial of a healthy brain ageing cognitive training program: effects on memory, mood, and sleep. Journal of Alzheimer's disease : JAD. 2015;44(4):1181-91.

9. Zhang H, Huntley J, Bhome R, Holmes B, Cahill J, Gould RL, et al. Effect of computerised cognitive training on cognitive outcomes in mild cognitive impairment: a systematic review and meta-analysis. BMJ Open. 2019;9(8):e027062.

10. Hill NT, Mowszowski L, Naismith SL, Chadwick VL, Valenzuela M, Lampit A. Computerized Cognitive Training in Older Adults With Mild Cognitive Impairment or Dementia: A Systematic Review and MetaAnalysis. The American journal of psychiatry. 2017;174(4):329-40.

11. Levine B, Stuss DT, Winocur G, Binns MA, Fahy L, Mandic M, et al. Cognitive rehabilitation in the elderly: effects on strategic behavior in relation to goal management. Journal of the International Neuropsychological Society : JINS. 2007;13(1):143-52.

12. Kurz A, Pohl C, Ramsenthaler M, Sorg C. Cognitive rehabilitation in patients with mild cognitive impairment. International journal of geriatric psychiatry. 2009;24(2):163-8.

13. Kinsella GJ, Mullaly E, Rand E, Ong B, Burton C, Price S, et al. Early intervention for mild cognitive impairment: a randomised controlled trial. Journal of neurology, neurosurgery, and psychiatry. 2009;80(7):730-6.

14. Norrie LM, Diamond K, Hickie IB, Rogers NL, Fearns S, Naismith SL. Can older "at risk" adults benefit from psychoeducation targeting healthy brain aging? International psychogeriatrics. 2011;23(3):413-24.

15. Rebok GW, Ball K, Guey LT, Jones RN, Kim HY, King JW, et al. Ten-year effects of the advanced cognitive training for independent and vital elderly cognitive training trial on cognition and everyday functioning in older adults. Journal of the American Geriatrics Society. 2014;62(1):16-24.

16. Rebok GW, Carlson MC, Langbaum JB. Training and maintaining memory abilities in healthy older adults: traditional and novel approaches. The journals of gerontology Series B, Psychological sciences and social sciences. 2007;62 Spec No 1:53-61. 
17. Bahar-Fuchs A, Clare L, Woods B. Cognitive training and cognitive rehabilitation for mild to moderate Alzheimer's disease and vascular dementia. The Cochrane database of systematic reviews. 2013(6):CD003260.

18. Mehrani I, Kochan NA, Ong MY, Crawford JD, Naismith SL, Sachdev PS. Organisational aspects and assessment practices of Australian memory clinics: an Australian Dementia Network (ADNeT) Survey. BMJ Open. 2021;11(2):e038624.

19. Dark F, Harris M, Gore-Jones V, Newman E, Whiteford H. Implementing cognitive remediation and social cognitive interaction training into standard psychosis care. BMC Health Serv Res. 2018;18(1):458.

20. John AP, Yeak K, Ayres H, Dragovic M. Successful implementation of a cognitive remediation program in everyday clinical practice for individuals living with schizophrenia. Psychiatr Rehabil J. 2017;40(1):87-93.

21. Cairns A, Dark F, Batts M. Implementing cognitive remediation therapy: lessons from two public mental health services. Australas Psychiatry. 2013;21(5):476-80.

22. McGurk SR, Mueser KT, Watkins MA, Dalton CM, Deutsch $\mathrm{H}$. The feasibility of implementing cognitive remediation for work in community based psychiatric rehabilitation programs. Psychiatr Rehabil J. 2017;40(1):79-86.

23. Hammar A, Semkovska M, Borgen IMH, Myklebost S, Ronold EH, Sveen T, et al. A pilot study of cognitive remediation in remitted major depressive disorder patients. Appl Neuropsychol Adult. 2020:1-11.

24. Eigenhuis E, Seldenrijk A, van Schaik A, Raes F, van Oppen P. Feasibility and Effectiveness of Memory Specificity Training in Depressed Outpatients: A Pilot Study. Clin Psychol Psychother. 2017;24(1):269-77.

25. Djabelkhir L, Wu YH, Vidal JS, Cristancho-Lacroix V, Marlats F, Lenoir H, et al. Computerized cognitive stimulation and engagement programs in older adults with mild cognitive impairment: comparing feasibility, acceptability, and cognitive and psychosocial effects. Clin Interv Aging. 2017;12:1967-75.

26. Glasgow RE, Vogt TM, Boles SM. Evaluating the public health impact of health promotion interventions: the RE-AIM framework. Am J Public Health. 1999;89(9):1322-7.

27. Stetler CB, Legro MW, Wallace CM, Bowman C, Guihan M, Hagedorn H, et al. The role of formative evaluation in implementation research and the QUERI experience. J Gen Intern Med. 2006;21 SuppI 2:S1-8.

28. Damschroder LJ, Aron DC, Keith RE, Kirsh SR, Alexander JA, Lowery JC. Fostering implementation of health services research findings into practice: a consolidated framework for advancing implementation science. Implement Sci. 2009;4:50.

29. Naismith SL, Diamond K, Carter PE, Norrie LM, Redoblado-Hodge MA, Lewis SJ, et al. Enhancing memory in late-life depression: the effects of a combined psychoeducation and cognitive training program. The American journal of geriatric psychiatry : official journal of the American Association for Geriatric Psychiatry. 2011;19(3):240-8. 
30. Medalia A, Freilich B. The Neuropsychological Educational Approach to Cognitive Remediation (NEAR) model: Practice principles and outcome studies. American Journal Psychiatric Rehabilitation. 2008;11:12343.

31. Randolph C, Tierney MC, Mohr E, Chase TN. The Repeatable Battery for the Assessment of Neuropsychological Status (RBANS): preliminary clinical validity. Journal of clinical and experimental neuropsychology. 1998;20(3):310-9.

32. Dubois B, Slachevsky A, Litvan I, Pillon B. The FAB: a Frontal Assessment Battery at bedside. Neurology. 2000;55(11):1621-6.

33. Yesavage JA, Adey M, Werner PD. Development of a geriatric behavioral self-assessment scale. Journal of the American Geriatrics Society. 1981;29(6):285-8.

34. Lovibond SHL, P. F. Manual for the Depression Anxiety Stress Scales. (2nd Ed.). Sydney: Psychology Foundation; 1995.

35. World Health Organization. Development of the World Health Organization WHOQOL-BREF quality of life assessment. The WHOQOL Group. Psychological medicine. 1998;28(3):551-8.

36. Ustun TB, Chatterji S, Kostanjsek N, Rehm J, Kennedy C, Epping-Jordan J, et al. Developing the World Health Organization Disability Assessment Schedule 2.0. Bulletin of the World Health Organization. 2010;88(11):815-23.

37. Crook TH, Feher EP, Larrabee GJ. Assessment of memory complaint in age-associated memory impairment: the MAC-Q. International psychogeriatrics. 1992;4(2):165-76.

38. Smith G, Della Sala S, Logie RH, Maylor EA. Prospective and retrospective memory in normal ageing and dementia: a questionnaire study. Memory. 2000;8(5):311-21.

39. Glasgow RE, Battaglia C, McCreight M, Ayele RA, Rabin BA. Making Implementation Science More Rapid: Use of the RE-AIM Framework for Mid-Course Adaptations Across Five Health Services Research Projects in the Veterans Health Administration. Front Public Health. 2020;8:194.

40. Linn BS, Linn MW, Gurel L. Cumulative illness rating scale. Journal of the American Geriatrics Society. 1968;16(5):622-6.

41. Sheehan DV, Lecrubier Y, Sheehan KH, Amorim P, Janavs J, Weiller E, et al. The Mini-International Neuropsychiatric Interview (M.I.N.I.): the development and validation of a structured diagnostic psychiatric interview for DSM-IV and ICD-10. The Journal of clinical psychiatry. 1998;59 Suppl 20:22-33;quiz 4-57.

42. Hamilton M. A rating scale for depression. Journal of neurology, neurosurgery, and psychiatry. 1960;23:56-62.

43. Wechsler D. Wechsler Adult Intelligence Scale - Fourth Edition. San Antonio, TX: Pearson; 2008. 
44. Strauss E. compendium of neuropsychological tests: Administration, norms, and commentary. New York: Oxford University Press; 2006.

45. Brandt J. The Hopkins Verbal Learning Test: Development of a new memory test with six equivalent forms. Clinical Neuropsychologist. 1991;5(2):125-42.

46. Delis DC, Kramer JH, Kaplan E, Holdnack J. Reliability and validity of the Delis-Kaplan Executive Function System: an update. Journal of the International Neuropsychological Society : JINS. 2004;10(2):3013.

47. Reitan RM. The relation of the trail making test to organic brain damage. Journal of consulting psychology. 1955;19(5):393-4.

48. Segal DL, June A, Payne M, Coolidge FL, Yochim B. Development and initial validation of a self-report assessment tool for anxiety among older adults: the Geriatric Anxiety Scale. Journal of anxiety disorders. 2010;24(7):709-14.

49. Buysse DJ, Reynolds CF, 3rd, Monk TH, Berman SR, Kupfer DJ. The Pittsburgh Sleep Quality Index: a new instrument for psychiatric practice and research. Psychiatry research. 1989;28(2):193-213.

50. Fillenbaum GG. Multidimensional Functional Assessment of Older Adults: The Duke Older Americans Resources and Services Procedures. . Hillsdale, NJ: Lawrence Erlbaum Associates; 1988.

51. World Health Organization. Wellbeing Measures in Primary Health Care/The Depcare Project. Copenhagen: WHO Regional Office for Europe; 1998.

52. Naismith SL, Redoblado-Hodge MA, Lewis SJ, Scott EM, Hickie IB. Cognitive training in affective disorders improves memory: a preliminary study using the NEAR approach. Journal of affective disorders. 2010;121(3):258-62.

53. Reeder C, Harris V, Pickles A, Patel A, Cella M, Wykes T. Does change in cognitive function predict change in costs of care for people with a schizophrenia diagnosis following cognitive remediation therapy? Schizophrenia bulletin. 2014;40(6):1472-81.

\section{Tables}

Table 1. Baseline and follow-up neuropsychological, mood and psychosocial data for Study 1 


\begin{tabular}{|c|c|c|c|c|c|c|c|}
\hline Outcome & $\mathrm{N}$ & $\begin{array}{l}\text { Mean (SD) } \\
\text { pre- } \\
\text { intervention }\end{array}$ & $\begin{array}{l}\text { Mean (SD) } \\
\text { post- } \\
\text { intervention }\end{array}$ & $\begin{array}{l}F, p- \\
\text { value }\end{array}$ & $95 \% \mathrm{Cl}$ & & $\begin{array}{l}\text { Partial } \\
\text { Eta } \\
\text { Squared }\end{array}$ \\
\hline RBANS List Learning ${ }^{a}$ & 24 & $8.42(2.59)$ & $9.46(2.90)$ & $\begin{array}{l}4.952 \\
0.036^{*}\end{array}$ & -2.01 & -0.07 & $0.177^{+++}$ \\
\hline RBANS Story Memory ${ }^{a}$ & 24 & $9.21(3.06)$ & $9.67(2.08)$ & $\begin{array}{l}0.580 \\
0.454\end{array}$ & -1.70 & 0.79 & 0.025 \\
\hline RBANS Figure Copy ${ }^{a}$ & 24 & $9.33(2.82)$ & $9.25(3.11)$ & $\begin{array}{l}0.026 \\
0.874\end{array}$ & -0.99 & 1.16 & 0.001 \\
\hline RBANS Line Orientation ${ }^{a}$ & 24 & $11.21(2.92)$ & $11.54(2.25)$ & $\begin{array}{l}0.594 \\
0.449\end{array}$ & -1.23 & 0.56 & $0.025^{+}$ \\
\hline RBANS Picture Naming a & 24 & $8.96(2.31)$ & $8.75(2.63)$ & $\begin{array}{l}0.162 \\
0.691\end{array}$ & -0.86 & 1.28 & 0.007 \\
\hline $\begin{array}{l}\text { RBANS Semantic } \\
\text { Fluency }{ }^{a}\end{array}$ & 24 & $8.50(3.02)$ & $10.42(2.32)$ & $\begin{array}{l}13.717 \\
0.001^{\star}\end{array}$ & -2.99 & -0.85 & $0.374^{+++}$ \\
\hline RBANS Digit Spana & 24 & $9.38(2.34)$ & $10.29(2.76)$ & $\begin{array}{l}2.321 \\
0.141\end{array}$ & -2.16 & 0.33 & $0.092^{++}$ \\
\hline RBANS Coding ${ }^{a}$ & 23 & $9.17(3.10)$ & $8.74(3.12)$ & $\begin{array}{l}0.905 \\
0.352\end{array}$ & -0.51 & 1.38 & $0.040^{+}$ \\
\hline RBANS List Recalla & 24 & $7.92(2.02)$ & $8.46(2.43)$ & $\begin{array}{l}1.929 \\
0.178\end{array}$ & -1.35 & 0.27 & $0.077^{++}$ \\
\hline RBANS List Recognition ${ }^{a}$ & 24 & 7.71(3.37) & $8.38(2.96)$ & $\begin{array}{l}1.813 \\
0.191\end{array}$ & -1.69 & 0.36 & $0.073^{++}$ \\
\hline RBANS Story Recalla & 24 & $8.46(3.46)$ & $9.29(3.01)$ & $\begin{array}{l}2.964 \\
0.099\end{array}$ & -1.83 & 0.17 & $0.114^{+++}$ \\
\hline RBANS Figure Recalla & 24 & $7.00(2.75)$ & $7.63(3.12)$ & $\begin{array}{l}1.404 \\
0.250\end{array}$ & -1.72 & 0.47 & $0.058^{+}$ \\
\hline $\begin{array}{l}\text { Frontal Assessment } \\
\text { Battery }{ }^{b}\end{array}$ & 24 & $15.08(2.19)$ & $15.75(2.64)$ & $\begin{array}{l}2.022 \\
0.168\end{array}$ & -1.64 & 0.30 & $0.081^{++}$ \\
\hline $\mathrm{HBAFAQ}^{\mathrm{b}}$ & 15 & $16.93(11.80)$ & $19.20(16.66)$ & $\begin{array}{l}0.190 \\
0.670\end{array}$ & -13.42 & 8.89 & $0.013^{+}$ \\
\hline WHO-DAS $2.0^{\mathrm{b}}$ & 16 & $44.63(19.80)$ & $41.81(17.55)$ & $\begin{array}{l}0.229 \\
0.716\end{array}$ & -7.88 & 13.51 & $0.186^{+++}$ \\
\hline MACQ $^{b}$ & 19 & $24.63(4.28)$ & $25.21(4.06)$ & $\begin{array}{l}0.236 \\
0.633\end{array}$ & -3.09 & 1.93 & $0.013^{+}$ \\
\hline $\mathrm{PRMQ}^{\mathrm{b}}$ & 14 & $44.43(9.61)$ & $40.14(11.06)$ & $\begin{array}{l}1.134 \\
0.306\end{array}$ & -4.41 & 12.98 & 0.080 \\
\hline $\begin{array}{l}\text { WHO- } \\
\text { QoL BREF Physical }\end{array}$ & 16 & $3.72(0.66)$ & $3.61(0.69)$ & $\begin{array}{l}0.292 \\
0.597\end{array}$ & -0.30 & 0.50 & $0.019^{+}$ \\
\hline
\end{tabular}


WHO-

$16 \quad 3.72(0.65)$

$3.44(0.65)$

5.696

0.03

0.53

$0.275^{+++}$

QoL BREF Psychological ${ }^{\text {b }}$

WHO-

16

3.89(0.78)

$5.65(7.31)$

0.878

0.364

$-5.79$

2.25

$0.055^{+}$

QoL BREF Relationships ${ }^{b}$

WHO-

$16 \quad 4.36(0.46)$

4.49(0.88)

0.261
0.617

$-0.63$

0.39

$0.017^{+}$

QoL BREF Enviroment ${ }^{b}$

\begin{tabular}{|c|c|c|c|c|c|c|c|}
\hline DASS Depression Scale ${ }^{b}$ & 23 & $4.57(4.35)$ & $3.17(2.33)$ & $\begin{array}{l}2.695 \\
0.115\end{array}$ & -0.37 & 3.15 & $0.109^{+++}$ \\
\hline DASS Anxiety Scale ${ }^{b}$ & 23 & $3.91(3.18)$ & $2.09(1.95)$ & $\begin{array}{l}9.516 \\
0.005^{\star}\end{array}$ & 0.60 & 3.05 & $0.302^{+++}$ \\
\hline DASS Stress Scale & 23 & $6.26(4.77)$ & $3.70(2.91$ & $\begin{array}{l}5.141 \\
0.034^{\star}\end{array}$ & 0.22 & 4.91 & $0.189^{+++}$ \\
\hline $\begin{array}{l}\text { Geriatric Depression } \\
\text { Scale }^{\mathrm{b}}\end{array}$ & 21 & $3.10(2.83)$ & $3.19(2.71)$ & $\begin{array}{l}0.024 \\
0.879\end{array}$ & -1.38 & 1.19 & 0.001 \\
\hline
\end{tabular}

a age and education scaled score; ${ }^{b}$ total raw score; * indicates statistical significance; ${ }^{+}$indicates small effect size; ${ }^{++}$indicates medium effect size; ${ }^{+++}$indicates large to very large effect size; $\mathrm{Cl}=$ confidence interval; DASS = Depression Anxiety Stress Scale; HBA-FAQ = Healthy Brain Ageing Frontal Assessment Questionnaire; MACQ = Memory Complaint Questionnaire; $\mathrm{PRMQ}=$ Prospective and Retrospective Memory Questionnaire; RBANS = Repeatable Battery for the Assessment of Neuropsychological Status; SD = standard deviation; WHO-DAS 2.0 = WHO Disability Assessment Scale 2.0; WHO-QoL BREF = WHO-Quality of Life BREF.

\section{Table 2. Baseline and follow-up neuropsychological, mood and psychosocial data for Study 2}




\begin{tabular}{|c|c|c|c|c|c|c|c|}
\hline Outcome & $\mathrm{N}$ & $\begin{array}{l}\text { Mean(SD) } \\
\text { pre- } \\
\text { intervention }\end{array}$ & $\begin{array}{l}\text { Mean(SD) } \\
\text { post- } \\
\text { intervention }\end{array}$ & $\begin{array}{l}F, p- \\
\text { value }\end{array}$ & \multicolumn{2}{|l|}{$95 \% \mathrm{Cl}$} & $\begin{array}{l}\text { Partial } \\
\text { Eta } \\
\text { Squared }\end{array}$ \\
\hline Similarities $^{a}$ & 34 & $10.41(2.71)$ & $10.71(3.01)$ & $\begin{array}{l}0.430 \\
0.521\end{array}$ & -1.22 & 0.63 & $0.013^{+}$ \\
\hline Coding ${ }^{a}$ & 33 & $9.42(2.42)$ & $9.58(2.82)$ & $\begin{array}{l}0.366 \\
0.549\end{array}$ & -0.66 & 0.36 & $0.011^{+}$ \\
\hline Logical Memory I ${ }^{a}$ & 34 & $9.12(3.02)$ & $9.62(3.02)$ & $\begin{array}{l}2.493 \\
0.124\end{array}$ & -1.14 & 0.14 & $0.070^{++}$ \\
\hline Logical Memory $\|^{a}$ & 34 & $9.09(3.15)$ & $9.35(3.14)$ & $\begin{array}{l}1.083 \\
0.306\end{array}$ & -0.78 & 0.25 & $0.032^{+}$ \\
\hline HVLT Total Learning ${ }^{b}$ & 33 & $0.04(1.09)$ & $0.26(1.25)$ & $\begin{array}{l}2.193 \\
0.148\end{array}$ & -0.52 & 0.08 & $0.064^{++}$ \\
\hline HVLT Delay ${ }^{b}$ & 32 & $-0.28(1.09)$ & $0.28(1.05)$ & $\begin{array}{l}\text { 10.167, } \\
0.003^{*}\end{array}$ & -0.92 & -0.20 & $0.247^{+++}$ \\
\hline $\begin{array}{l}\text { HVLT Discrimination } \\
\text { Index }\end{array}$ & 31 & $-0.5(1.12)$ & $-0.32(1.5)$ & $\begin{array}{l}0.946 \\
0.339\end{array}$ & -0.56 & 0.20 & $0.031^{+}$ \\
\hline RBANS Figure Copy ${ }^{c}$ & 31 & $10.77(2.7)$ & 10.1(3.3) & $\begin{array}{l}1.899 \\
0.178\end{array}$ & -0.33 & 1.68 & $0.060^{++}$ \\
\hline RBANS Figure Recallc & 27 & $8.48(2.69)$ & $10.04(3.23)$ & $\begin{array}{l}\text { 14.315, } \\
0.001 *\end{array}$ & -2.40 & -0.71 & $0.355^{+++}$ \\
\hline Letter Fluency Total ${ }^{\mathrm{b}}$ & 33 & $0.17(1.24)$ & $0.3(1.12)$ & $\begin{array}{l}0.954 \\
0.336\end{array}$ & -0.41 & 0.14 & $0.029^{+}$ \\
\hline Semantic Fluency Total ${ }^{b}$ & 32 & $-0.04(1.4)$ & $-0.44(1.48)$ & $\begin{array}{l}3.690 \\
0.064\end{array}$ & -0.03 & 0.84 & $0.106^{++}$ \\
\hline $\begin{array}{l}\text { Colour Word Interference } \\
\text { Test Naming }^{\text {a }}\end{array}$ & 28 & $8.25(3.71)$ & 8.93(3.33) & $\begin{array}{l}1.722 \\
0.200\end{array}$ & -1.74 & 0.38 & $0.060^{++}$ \\
\hline $\begin{array}{l}\text { Colour Word Interference } \\
\text { Test Reading a }\end{array}$ & 28 & $10.36(2.84)$ & $10.18(2.74)$ & $\begin{array}{l}0.165 \\
0.688\end{array}$ & -0.72 & 1.08 & 0.006 \\
\hline $\begin{array}{l}\text { Colour Word Interference } \\
\text { Test Inhibition a }\end{array}$ & 28 & $8.25(4.03)$ & $9(3.91)$ & $\begin{array}{l}1.460 \\
0.237\end{array}$ & -2.02 & 0.52 & $0.051^{+}$ \\
\hline $\begin{array}{l}\text { Colour Word Interference } \\
\text { Test Inhibition } \\
\text { Switching }^{\text {a }}\end{array}$ & 26 & $9.04(4.01)$ & $8.54(4.54)$ & $\begin{array}{l}0.705 \\
0.409\end{array}$ & -0.73 & 1.73 & $0.027^{+}$ \\
\hline Trail Making Test $A^{b}$ & 4 & $-1.68(2.16)$ & $-1.11(0.93)$ & $\begin{array}{l}0.724 \\
0.457\end{array}$ & -2.71 & 1.57 & $0.194^{+++}$ \\
\hline Trail Making Test $\mathrm{B}^{\mathrm{b}}$ & 4 & $-2.43(2.66)$ & $-5.71(10.44)$ & $\begin{array}{l}0.633 \\
0.484\end{array}$ & -9.84 & 16.40 & $0.174^{+++}$ \\
\hline $\begin{array}{l}\text { Geriatric Depression } \\
\text { Scale }^{d}\end{array}$ & 42 & $4.00(3.46)$ & $3.88(3.19)$ & $\begin{array}{l}0.060 \\
0.808\end{array}$ & -0.86 & 1.10 & 0.001 \\
\hline
\end{tabular}




\begin{tabular}{|c|c|c|c|c|c|c|c|}
\hline DASS Depression Scale ${ }^{d}$ & 41 & $4.12(4.60)$ & $3.76(3.58)$ & $\begin{array}{l}0.523 \\
0.474\end{array}$ & -0.66 & 1.39 & $0.013^{+}$ \\
\hline DASS Anxiety Scale ${ }^{d}$ & 41 & $3.76(3.61)$ & 2.83(3.02) & $\begin{array}{l}3.905 \\
0.55\end{array}$ & -0.02 & 1.87 & $0.089^{++}$ \\
\hline DASS Stress Scale ${ }^{d}$ & 41 & $4.59(4.39)$ & $3.98(4.60)$ & $\begin{array}{l}2.104 \\
0.155\end{array}$ & -0.24 & 1.46 & $0.050^{+}$ \\
\hline $\begin{array}{l}\text { Pittsburgh Sleep } \\
\text { Inventory Questionnaire }\end{array}$ & 37 & $6.16(3.10)$ & $6.49(4.21)$ & $\begin{array}{l}0.347 \\
0.560\end{array}$ & -1.44 & 0.79 & $0.010^{+}$ \\
\hline $\begin{array}{l}\text { WHO- } \\
\text { QoL BREF Physical }\end{array}$ & 40 & $63.60(13.51)$ & $62.05(14.03)$ & $\begin{array}{l}0.690 \\
0.411\end{array}$ & -2.22 & 5.32 & $0.017^{+}$ \\
\hline $\begin{array}{l}\text { WHO- } \\
\text { QoL BREF Psychological }\end{array}$ & 40 & $61.50(14.60)$ & $20.85(2.81)$ & $\begin{array}{l}0.053 \\
0.819\end{array}$ & 36.52 & 44.78 & 0.001 \\
\hline $\begin{array}{l}\text { WHO- } \\
\text { QoL BREF Relationships } \\
\text { d }\end{array}$ & 40 & 65.75(22.59) & $62.85(19.48)$ & $\begin{array}{l}0.928 \\
0.341\end{array}$ & -3.19 & 8.99 & $0.023^{+}$ \\
\hline $\begin{array}{l}\text { WHO- } \\
\text { QoL BREF Environmen }\end{array}$ & 40 & 79.38(20.05) & $82.00(13.73)$ & $\begin{array}{l}1.676 \\
0.203\end{array}$ & -6.73 & 1.48 & $0.041^{+}$ \\
\hline OARS MFAQ_IADL ${ }^{d}$ & 27 & $12.56(2.14)$ & $13.04(1.34)$ & $\begin{array}{l}1.876 \\
0.182\end{array}$ & -1.20 & 0.24 & $0.067^{++}$ \\
\hline OARS MFAQ physicald & 28 & $13.29(1.01)$ & $13.46(1.32)$ & $\begin{array}{l}1.000 \\
0.326\end{array}$ & -0.54 & 0.19 & $0.036^{+}$ \\
\hline OARS_MFAQ Q.101 & 26 & $2.46(1.07)$ & $2.15(1.12)$ & $\begin{array}{l}3.150 \\
0.088\end{array}$ & -0.05 & 0.66 & $0.112^{++}$ \\
\hline GAS Somatic $^{d}$ & 9 & $4.33(2.18)$ & $4.00(2.50)$ & $\begin{array}{l}0.148 \\
0.710\end{array}$ & -1.66 & 2.33 & $0.018^{+}$ \\
\hline GAS Cognitive ${ }^{d}$ & 9 & $2.44(3.81)$ & $2.78(2.28)$ & $\begin{array}{l}0.138 \\
0.720\end{array}$ & -2.40 & 1.74 & $0.017^{+}$ \\
\hline GAS Affective ${ }^{d}$ & 10 & $4.00(5.79)$ & $3.20(3.19)$ & $\begin{array}{l}0.298 \\
0.599\end{array}$ & -2.52 & 4.12 & $0.032^{+}$ \\
\hline Cognitive Stimulation & 8 & $35.50(5.13)$ & $36.13(4.73)$ & $\begin{array}{l}0.223 \\
0.651\end{array}$ & -3.75 & 2.50 & $0.031^{+}$ \\
\hline $\begin{array}{l}\text { WHO Welling Being } \\
\text { Index }\end{array}$ & 10 & $12.80(5.65)$ & $14.20(6.41)$ & $\begin{array}{l}0.766 \\
0.404\end{array}$ & -5.02 & 2.22 & $0.078^{++}$ \\
\hline HBA-FAQ & 9 & $19.78(13.87)$ & $14.78(8.61)$ & $\begin{array}{l}1.610 \\
0.240\end{array}$ & -4.09 & 14.09 & $0.168^{+++}$ \\
\hline
\end{tabular}

a age corrected scaled score; ${ }^{b}$ age and educated corrected $z$-score; ${ }^{c}=$ age and educated corrected scaled score; ${ }^{d}=$ total raw score; ${ }^{*}$ indicates statistical significance; ${ }^{+}$indicates small effect size; ${ }^{++}$indicates medium effect size; ${ }^{+++}$indicates large to very large effect size; $\mathrm{Cl}=$ confidence interval; DASS = Depression Anxiety Stress Scale; HBA-FAQ = Healthy Brain Ageing Frontal Assessment Questionnaire; HVLT = Hopkins Verbal Learning Test; IADL = instrumental activities of daily activities; MFAQ = Multidimensional Functional 
Assessment Questionnaire; RBANS = Repeatable Battery for the Assessment of Neuropsychological Status; SD = standard deviation; WHO-QoL BREF = WHO Quality of Life.

\section{Figures}

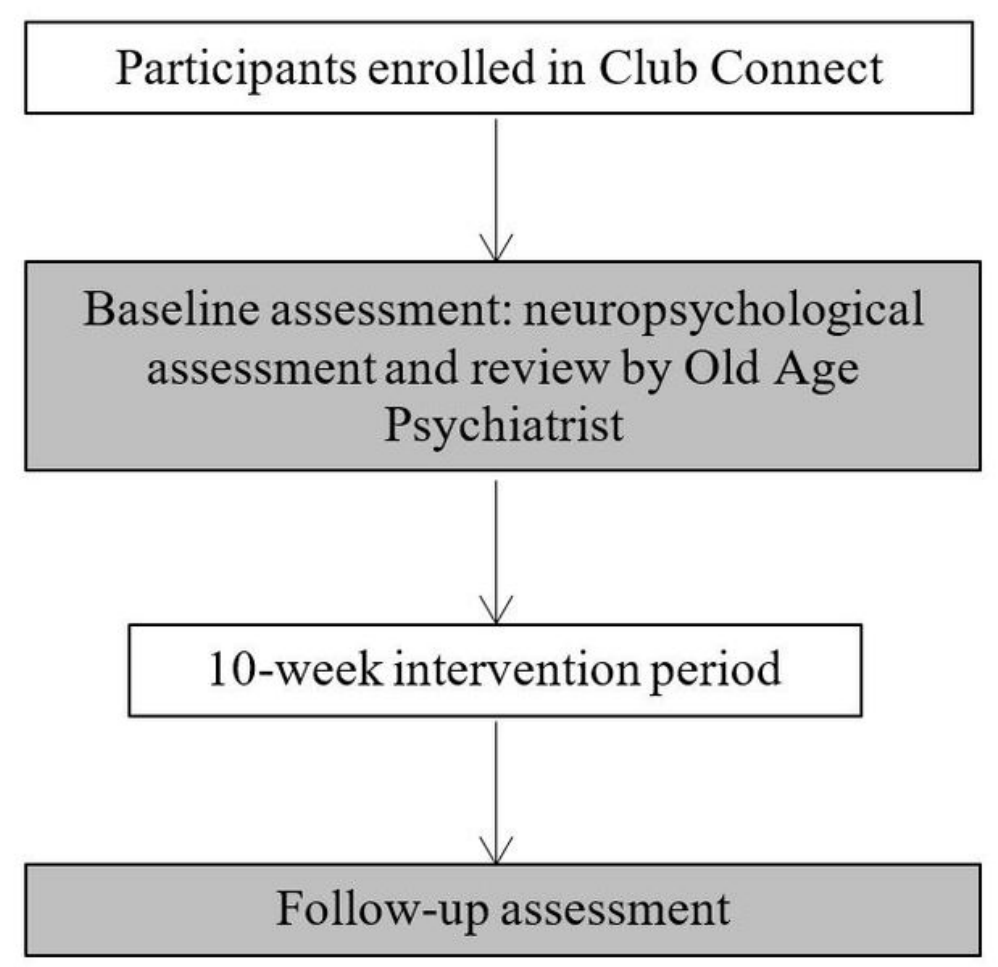

Time

1-2 weeks

3-12 weeks

13-14 weeks

\section{Figure 1}

Participant flow for Studies 1 and 2

\section{Supplementary Files}

This is a list of supplementary files associated with this preprint. Click to download.

- WoolfAdditionalFile1.docx

- WoolfAdditionalFile2.docx 